\title{
Efficient Variability Analysis of Electromagnetic Systems via Polynomial Chaos and Model Order Reduction
}

\author{
Domenico Spina, Francesco Ferranti, Member, IEEE, Giulio Antonini, Senior Member, IEEE, Tom \\ Dhaene, Senior Member, IEEE, Luc Knockaert, Senior Member, IEEE
}

\begin{abstract}
We present a novel technique to perform the model order reduction of multiport systems under the effect of statistical variability of geometrical or electrical parameters. The proposed approach combines a deterministic model order reduction phase with the use of the Polynomial Chaos expansion to perform the variability analysis of the system under study very efficiently. The combination of model order reduction and Polynomial Chaos techniques generates a final reduced-order model able to accurately perform stochastic computations and variability analysis. The novel proposed method guarantees a high-degree of flexibility, since different model order reduction schemes can be used and different types of modern electrical systems (e.g. filters, connectors) can be modeled. The accuracy and efficiency of the proposed approach is verified by means of two numerical examples and compared with other existing variability analysis techniques.
\end{abstract}

Index Terms-Multiport systems, variability analysis, polynomial chaos, model order reduction.

\section{INTRODUCTION}

Recently, the necessity of employing efficient techniques to perform the variability analysis (VA) of the modern integrated circuits has become evident. The Monte Carlo (MC) -based techniques, that represent the standard for the VA due to their accuracy and ease of implementation, have the drawback of requiring a very high computational cost. In particular, the application of the MC method to complex high-speed systems analyzed by means of electromagnetic (EM) methods [1][3] is unfeasible. Indeed, EM methods usually produce very large systems of equations which are expensive to solve, and the use of the MC analysis would lead to an extremely high computational cost.

In this scenario, a reliable alternative to MC-based methods is represented by techniques based on the Polynomial Chaos (PC) expansion [4]-[8], which describes a stochastic process using a base of orthogonal polynomial functions with suitable coefficients. The resulting polynomial model allows to efficiently perform the VA with good accuracy, at the cost of the calculation of an augmented system [4]-[8]. Unfortunately,

Domenico Spina, Francesco Ferranti, Tom Dhaene and Luc Knockaert are with the Department of Information Technology, Internet Based Communication Networks and Services (IBCN), Ghent University - IMinds, Gaston Crommenlaan 8 Bus 201, B-9050 Gent, Belgium, email: \{domenico.spina, francesco.ferranti, tom.dhaene, luc.knockaert $\} @$ intec.ugent.be;

Giulio Antonini is with the UAq EMC Laboratory, Dipartimento di Ingegneria Industriale e dell'Informazione e di Economia, Università degli Studi dell'Aquila, Via G. Gronchi 18, 67100, L'Aquila, Italy, phone: +390862434462, email: giulio.antonini@univaq.it. the application of the PC expansion to systems described by a large numbers of equations, such as the ones resulting by the use of EM methods, is not trivial due to the need of calculating an augmented system.

Different techniques [9]-[13] have proposed PC-based methodologies for the VA of systems described by a large numbers of equations based on combinations of a PC expansion on the original system matrices and MOR techniques. Indeed, the MOR techniques allow to reduce the complexity of large scale models and, therefore, the computational cost of the simulations [14]-[16]. Recently, the techniques [11][13] propose a more general PC-based method for the VA of large scale systems described by Helmholtz equations. The recently proposed methods described in [11]-[13] are based on the following steps:

- the evaluation of the original large system of equations over a discrete set of points in the stochastic space, chosen using a Smolyak grid [17]-[19];

- the use of a deterministic MOR technique for each system of equations to generate the corresponding projection matrices;

- the calculation of the PC expansion of the original large system matrices and of the projection matrices;

- the computation of the PC coefficients of the reduced system using congruence transformations;

- the calculation of a PC-based augmented system.

Despite their accuracy and efficiency with respect to the MCbased methods, the techniques [11]-[13] can be expensive both in terms of memory and computational time, since they require the calculation of a PC-based model of the original large scale equations and of the projection matrices.

We propose in this paper a novel method that follows these steps:

- the evaluation of the original large system of equations over a discrete set of points in the stochastic space, chosen using a regular grid;

- the calculation of a corresponding set of reduced order models with common order using a common compact projection matrix, following the technique [20];

- the computation of the PC expansion of the reduced models;

- the calculation of a PC-based augmented system.

This new proposed technique is able to overcome the previously mentioned limitations by first calculating a set of 
reduced order models with common order using a common compact projection matrix and then computing the PC expansion of the reduced models.

This paper is structured as follows. First, an overview of PC theory is given in Section II. The proposed stochastic model order reduction technique is described in Section III, while its validation is performed in Section IV by means of two pertinent numerical examples. Conclusions are summed up in Section V.

\section{Polynomial ChaOS PROPERTIES}

A stochastic process $H$ with finite variance can be expressed by means of the PC expansion as [8]

$$
H=\sum_{i=0}^{\infty} \alpha_{i} \varphi_{i}(\boldsymbol{\xi})
$$

where the terms $\alpha_{i}$ are called PC coefficients and $\varphi_{i}(\boldsymbol{\xi})$ are the corresponding orthogonal polynomials that depend on the normalized random variables in the vector $\boldsymbol{\xi}$. The polynomials $\varphi_{i}(\boldsymbol{\xi})$, also called basis functions, satisfy the following orthogonality condition [6]

$$
<\varphi_{i}(\boldsymbol{\xi}), \varphi_{j}(\boldsymbol{\xi})>=\int_{\boldsymbol{\Omega}} \varphi_{i}(\boldsymbol{\xi}) \varphi_{j}(\boldsymbol{\xi}) W(\boldsymbol{\xi}) d \boldsymbol{\xi}=a_{i} \delta_{i j}
$$

where $a_{i}$ are positive numbers, $\delta_{i j}$ is the Kronecker delta and $W(\boldsymbol{\xi})$ is a probability measure with support $\boldsymbol{\Omega}$.

Obviously, (1) must be truncated up to a finite number of polynomials $M+1$ to be used for practical applications. In particular, if the random variables $\boldsymbol{\xi}$ are independent, it is possible to prove that

$$
M+1=\frac{(N+P) !}{N ! P !}
$$

where $N$ is the number of random variables $\xi_{i}$ in the vector $\xi$ and $P$ is the maximum order of the polynomials used in the truncated PC expansion. Indeed, for independent random variables the probability measure $W(\boldsymbol{\xi})$ can be written as

$$
W(\boldsymbol{\xi})=\prod_{i=1}^{N} W_{i}\left(\xi_{i}\right)
$$

since the global probability density function (PDF) is the product of the PDFs of the single random variables. Consequently, the corresponding orthogonal polynomials can be expressed as product combination of the basis functions corresponding to each random variable $\xi_{i}$ as

$\varphi_{j}(\boldsymbol{\xi})=\prod_{k=1}^{N} \phi_{i_{k}}\left(\xi_{k}\right) \quad$ with $\quad \sum_{k=1}^{N} i_{k} \leq P \quad$ and $\quad 0 \leq j \leq M$

Furthermore, if the random variables $\boldsymbol{\xi}$ have specific PDFs (i.e. Gaussian, Uniform, Beta distribution), the corresponding basis functions are the polynomials of the Wiener-Askey scheme [4]. Indeed, the choice of these polynomials guarantee an exponential convergence rate of the PC expansion, since the associated probability measure $W(\boldsymbol{\xi})$ corresponds to the PDF of the random variable $\boldsymbol{\xi}$, when placed in a standard form [4], [6]. Finally, in [6] it is presented a numerical method to calculate the basis functions that guarantee an exponential convergence rate for independent random variables with arbitrary distributions.

In the more general case of correlated random variables with arbitrary PDFs, the basis functions can be calculated following the approach described in [5]-[7]. In this case, the $\mathrm{PC}$ expansion convergence rate may not be exponential, since a variable transformation, such as the Nataf transformation [21] or the Karhunen-Loéve expansion [22] is needed to obtain the decorrelation.

Once the $M+1$ basis functions $\varphi_{i}(\boldsymbol{\xi})$ are calculated, (1) can be truncated as

$$
H \approx \sum_{i=0}^{M} \alpha_{i} \varphi_{i}(\boldsymbol{\xi})
$$

where the only unknown terms are the PC coefficients $\alpha_{i}$, that can be calculated following one of the two main methods described in the literature: the spectral projection and the linear regression technique [7].

The most interesting characteristic of the PC expansion is the analytical representation of the system variability: complex stochastic functions of $H$, such as the PDF, can be efficiently calculated following standard analytical formulas or numerical schemes [23], while the mean $\mu$ and the variance $\sigma^{2}$ of $H$ can be expressed as [7]

$$
\begin{aligned}
\mu & =\alpha_{0} \\
\sigma^{2} & =\sum_{i=1}^{M} \alpha_{i}^{2}<\varphi_{i}(\boldsymbol{\xi}), \varphi_{i}(\boldsymbol{\xi})>
\end{aligned}
$$

Finally, if the stochastic process is written in a matrix form $\boldsymbol{H}$, the corresponding PC expansion is

$$
\boldsymbol{H} \approx \sum_{i=0}^{M} \boldsymbol{\alpha}_{i} \varphi_{i}(\boldsymbol{\xi})
$$

where the terms $\boldsymbol{\alpha}_{i}$ are matrices of PC coefficients, corresponding to the $i$-th polynomial basis, calculated for each entry of $\boldsymbol{H}$. For an extensive reference to polynomial chaos theory, the reader may consult [4]-[8].

\section{Stochastic Model ORder Reduction}

The proposed technique aims to perform the VA of a generic multiport system represented by a descriptor state-space form as

$$
\begin{aligned}
(s \boldsymbol{C}(\boldsymbol{\xi})+\boldsymbol{G}(\boldsymbol{\xi})) \boldsymbol{X}(s, \boldsymbol{\xi}) & =\boldsymbol{B}(\boldsymbol{\xi}) \\
\boldsymbol{H}(s, \boldsymbol{\xi}) & =\boldsymbol{L}^{T}(\boldsymbol{\xi}) \boldsymbol{X}(s, \boldsymbol{\xi})
\end{aligned}
$$

where the descriptor state-space matrices $\boldsymbol{C}, \boldsymbol{G}, \boldsymbol{B}, \boldsymbol{L}$, that depend on a vector of random variables $\boldsymbol{\xi}$, are large matrices calculated by an EM method, such as the Partial Element Equivalent Circuit (PEEC) technique [2]. The superscript $T$ represents the matrix transpose. The dimensionality of descriptor state-space matrices in (10) and (11) is $C \in \mathbb{R}^{Z \times Z}, \boldsymbol{G} \in$ $\mathbb{R}^{Z \times Z}, \boldsymbol{B} \in \mathbb{R}^{Z \times N_{p}}, \boldsymbol{L} \in \mathbb{R}^{Z \times N_{p}}$, where $Z$ is the number of state-vector unknowns and depends on the particular EM method used to compute (10) and (11), and $N_{p}$ represent the 
number of ports of the system. In some recent contributions [24]-[26], it is proven that is possible to calculate efficiently the PC expansion of the system starting from the PC expansion of the corresponding model (state-space models in [24] and transmission line models in [25], [26]). Theoretically, a similar approach could be used for systems described by equations (10) and (11). Indeed, using the PC expansion (9) to express the state-space matrices, the state-vector and the output in equations (10) and (11) yields

$$
\begin{aligned}
& s \sum_{i=0}^{M} \sum_{j=0}^{M} \boldsymbol{C}_{i} \boldsymbol{X}_{j}(s) \varphi_{i}(\boldsymbol{\xi}) \varphi_{j}(\boldsymbol{\xi})= \\
& \quad-\sum_{i=0}^{M} \sum_{j=0}^{M} \boldsymbol{G}_{i} \boldsymbol{X}_{j}(s) \varphi_{i}(\boldsymbol{\xi}) \varphi_{j}(\boldsymbol{\xi})+\sum_{i=0}^{M} \boldsymbol{B}_{i} \varphi_{i}(\boldsymbol{\xi}) \\
& \sum_{j=0}^{M} \boldsymbol{H}_{j}(s) \varphi_{j}(\boldsymbol{\xi})=\sum_{i=0}^{M} \sum_{j=0}^{M} \boldsymbol{L}_{i}^{T} \boldsymbol{X}_{j}(s) \varphi_{i}(\boldsymbol{\xi}) \varphi_{j}(\boldsymbol{\xi})
\end{aligned}
$$

Let us assume for the moment that the PC expansion of the state-space matrices in equations (12) and (13) is already calculated. Therefore, the only unknowns are the PC coefficient matrices of the state vector $\boldsymbol{X}_{j}$ and of the transfer function $\boldsymbol{H}_{j}$.

The desired PC coefficient matrices can be calculated by projecting the equations (12) and (13) on each basis function $\varphi_{p}(\boldsymbol{\xi})$ for $p=0, \ldots, M$ (this procedure is referred as Galerkin projections [4], [25] in the PC theory). Indeed, projecting (12) on the basis function $\varphi_{p}(\boldsymbol{\xi})$ yields

$$
\begin{aligned}
& s \sum_{i=0}^{M} \sum_{j=0}^{M} \boldsymbol{C}_{i} \boldsymbol{X}_{j}(s)<\varphi_{i} \varphi_{j}, \varphi_{p}>= \\
& -\sum_{i=0}^{M} \sum_{j=0}^{M} \boldsymbol{G}_{i} \boldsymbol{X}_{j}(s)<\varphi_{i} \varphi_{j}, \varphi_{p}>+\sum_{i=0}^{M} \boldsymbol{B}_{i}<\varphi_{i}, \varphi_{p}>
\end{aligned}
$$

where the explicit dependency on the vector $\boldsymbol{\xi}$ is omitted, for the sake of clarity. Repeating this operation for $p=0, \ldots, M$ gives a frequency dependent linear system of the form

$$
\boldsymbol{\Phi}_{X} \boldsymbol{X}_{\alpha}=\boldsymbol{B}_{\alpha}
$$

where $\boldsymbol{\Phi}_{X} \in \mathbb{R}^{(M+1) Z \times(M+1) Z}, \boldsymbol{X}_{\alpha} \in \mathbb{R}^{(M+1) Z \times N_{p}}$, and $\boldsymbol{B}_{\alpha} \in \mathbb{R}^{(M+1) Z \times N_{p}}$.

To describe how it is possible to obtain equation (15), let us assume for simplicity that one random variable and two basis functions are used for the PC expansion. In this simplified case, equation (14) can be rewritten as

$$
\begin{array}{r}
\left(s \boldsymbol{C}_{0}+\boldsymbol{G}_{0}\right) \boldsymbol{X}_{0} \varphi_{0} \varphi_{0}+\left(s \boldsymbol{C}_{1}+\boldsymbol{G}_{1}\right) \boldsymbol{X}_{0} \varphi_{1} \varphi_{0} \\
+\left(s \boldsymbol{C}_{0}+\boldsymbol{G}_{0}\right) \boldsymbol{X}_{1} \varphi_{0} \varphi_{1}+\left(s \boldsymbol{C}_{1}+\boldsymbol{G}_{1}\right) \boldsymbol{X}_{1} \varphi_{1} \varphi_{1}= \\
\boldsymbol{B}_{0} \varphi_{0}+\boldsymbol{B}_{1} \varphi_{1}
\end{array}
$$

Now, thanks to the orthogonality relation (2), the projection of (16) onto the basis function $\varphi_{0}$ gives

$$
\begin{gathered}
\boldsymbol{E}_{0}(s) \boldsymbol{X}_{0}<\varphi_{0} \varphi_{0}, \varphi_{0}>+\boldsymbol{E}_{1}(s) \boldsymbol{X}_{0}<\varphi_{1} \varphi_{0}, \varphi_{0}> \\
+\boldsymbol{E}_{0}(s) \boldsymbol{X}_{1}<\varphi_{0} \varphi_{1}, \varphi_{0}>+\boldsymbol{E}_{1}(s) \boldsymbol{X}_{1}<\varphi_{1} \varphi_{1}, \varphi_{0}>= \\
\boldsymbol{B}_{0}<\varphi_{0}, \varphi_{0}>
\end{gathered}
$$

where $\boldsymbol{E}_{i}(s)=\left(s \boldsymbol{C}_{i}+\boldsymbol{G}_{i}\right)$ for $i=0,1$. The projection of (16) onto the basis function $\varphi_{1}$ yields

$$
\begin{gathered}
\boldsymbol{E}_{0}(s) \boldsymbol{X}_{0}<\varphi_{0} \varphi_{0}, \varphi_{1}>+\boldsymbol{E}_{1}(s) \boldsymbol{X}_{0}<\varphi_{1} \varphi_{0}, \varphi_{1}> \\
+\boldsymbol{E}_{0}(s) \boldsymbol{X}_{1}<\varphi_{0} \varphi_{1}, \varphi_{1}>+\boldsymbol{E}_{1}(s) \boldsymbol{X}_{1}<\varphi_{1} \varphi_{1}, \varphi_{1}>= \\
\boldsymbol{B}_{1}<\varphi_{1}, \varphi_{1}>
\end{gathered}
$$

Next, equations (17) and (18) can be rewritten in the form (15) as

$$
\left(\begin{array}{ll}
\boldsymbol{\Phi}_{X 00} & \boldsymbol{\Phi}_{X 01} \\
\boldsymbol{\Phi}_{X 10} & \boldsymbol{\Phi}_{X 11}
\end{array}\right)\left(\begin{array}{l}
\boldsymbol{X}_{0} \\
\boldsymbol{X}_{1}
\end{array}\right)=\left(\begin{array}{l}
\boldsymbol{B}_{0} \\
\boldsymbol{B}_{1}
\end{array}\right)
$$

where

$$
\begin{aligned}
& \boldsymbol{\Phi}_{X 00}=\boldsymbol{E}_{0}(s) \frac{<\varphi_{0} \varphi_{0}, \varphi_{0}>}{<\varphi_{0}, \varphi_{0}>}+\boldsymbol{E}_{1}(s) \frac{<\varphi_{1} \varphi_{0}, \varphi_{0}>}{<\varphi_{0}, \varphi_{0}>} \\
& \boldsymbol{\Phi}_{X 01}=\boldsymbol{E}_{0}(s) \frac{<\varphi_{0} \varphi_{1}, \varphi_{0}>}{<\varphi_{0}, \varphi_{0}>}+\boldsymbol{E}_{1}(s) \frac{<\varphi_{1} \varphi_{1}, \varphi_{0}>}{<\varphi_{0}, \varphi_{0}>} \\
& \boldsymbol{\Phi}_{X 10}=\boldsymbol{E}_{0}(s) \frac{\left\langle\varphi_{0} \varphi_{0}, \varphi_{1}>\right.}{<\varphi_{1}, \varphi_{1}>}+\boldsymbol{E}_{1}(s) \frac{<\varphi_{1} \varphi_{0}, \varphi_{1}>}{<\varphi_{1}, \varphi_{1}>} \\
& \boldsymbol{\Phi}_{X 11}=\boldsymbol{E}_{0}(s) \frac{<\varphi_{0} \varphi_{1}, \varphi_{1}>}{<\varphi_{1}, \varphi_{1}>}+\boldsymbol{E}_{1}(s) \frac{<\varphi_{1} \varphi_{1}, \varphi_{1}>}{<\varphi_{1}, \varphi_{1}>}
\end{aligned}
$$

The frequency dependent system (19) can now be solved for each frequency of interest, upon calculation of the scalar products in (20). Finally, the PC coefficients of the output $\boldsymbol{H}_{j}(s)$ can be computed using the PC coefficients of state vector $\boldsymbol{X}_{j}(s)$. Indeed, the projection of equation (13) onto the basis functions $\varphi_{p}(\boldsymbol{\xi}), p=0, \ldots, M$, leads to

$$
\boldsymbol{H}_{p}(s)=\sum_{i=0}^{M} \sum_{j=0}^{M} \boldsymbol{L}_{i}^{T} \boldsymbol{X}_{j}(s) \frac{<\varphi_{i}(\boldsymbol{\xi}) \varphi_{j}(\boldsymbol{\xi}), \varphi_{p}(\boldsymbol{\xi})>}{<\varphi_{p}(\boldsymbol{\xi}), \varphi_{p}(\boldsymbol{\xi})>}
$$

where all the scalar products were already calculated in order to build the matrix $\boldsymbol{\Phi}_{X}$.

However, the approach described above can not be efficiently used for systems described by equations (10) and (11). The calculation of the PC expansion (9) for the large matrices $\boldsymbol{C}, \boldsymbol{G}, \boldsymbol{B}, \boldsymbol{L}$, would be very expensive both in terms of memory and computational time, since each corresponding PC coefficient matrix would have the same dimension of the original matrix. Furthermore, the PC expansion of the matrices $\boldsymbol{C}, \boldsymbol{G}, \boldsymbol{B}, \boldsymbol{L}$, would lead to an augmented system in the form (15) of such a high dimension that the computational cost required to solve it may compromise the efficiency of the PC expansion with respect to the MC analysis.

The previously developed techniques [11]-[13] partially solve these issues for systems described by Helmholtz equations. Indeed, in [11]-[13] it is proposed to evaluate first the original large system of equations over a discrete set of points in the design space and then employ a deterministic MOR technique for each system of equations to generate the corresponding projection matrix. Then, it is calculated a PC model of the original large system matrices and of the projection matrix. It is important to notice that, following the approaches described in [11]-[13], all the projection matrices calculated for the initial discrete set of points must have common dimensions, otherwise it is not possible to compute the corresponding PC model of the projection matrix via numerical integration (see, for example, equation (33) in [13]). Indeed, it is not possible to calculate a summation of 
matrices with different dimensions. Finally, it is performed the computation of the PC coefficients of the reduced system that leads to an augmented system in a form similar to (15), but the overall dimension of this augmented system is drastically reduced. A compact PC expansion of the original system can be now calculated by employing standard deterministic techniques to solve the obtained augmented system. This approach is accurate and efficient with respect to the MC analysis (that has an extremely high computational cost, since it requires a huge number of simulation of the original large scale model). Furthermore, the techniques [11]-[13] offer the possibility to use different MOR techniques to calculate the corresponding reduced order PC models. However, the techniques [11]-[13] can be expensive both in terms of memory and computational time since it is required

- to calculate a PC model of the original large scale equations;

- to calculate a PC model of the projection matrices;

The novel method described in this paper is able to overcome these limitations by first calculating a set of reduced order models with common order using a common compact projection matrix, following the technique [20], and then computing the PC expansion of the reduced system. In particular, the method described in [20] is implemented using a worstcase choice for the estimation of the reduced model order and a global approach to build the common compact projection matrix. In the following, we will describe in details the novel proposed method.

Note that, fixing the value of the random variables $\boldsymbol{\xi}=\overline{\boldsymbol{\xi}}$ in equations (10) and (11) yields

$$
\begin{aligned}
(s \boldsymbol{C}(\overline{\boldsymbol{\xi}})+\boldsymbol{G}(\overline{\boldsymbol{\xi}})) \boldsymbol{X}(s, \overline{\boldsymbol{\xi}}) & =\boldsymbol{B}(\overline{\boldsymbol{\xi}}) \\
\boldsymbol{H}(s, \overline{\boldsymbol{\xi}}) & =\boldsymbol{L}^{T}(\overline{\boldsymbol{\xi}}) \boldsymbol{X}(s, \overline{\boldsymbol{\xi}})
\end{aligned}
$$

Now, it is possible to calculate an equivalent reduced order models as

$$
\begin{aligned}
(s \widehat{\boldsymbol{C}}(\overline{\boldsymbol{\xi}})+\widehat{\boldsymbol{G}}(\overline{\boldsymbol{\xi}})) \widehat{\boldsymbol{X}}(s, \overline{\boldsymbol{\xi}}) & =\widehat{\boldsymbol{B}}(\overline{\boldsymbol{\xi}}) \\
\boldsymbol{H}(s, \overline{\boldsymbol{\xi}}) & =\widehat{\boldsymbol{L}}^{T}(\overline{\boldsymbol{\xi}}) \widehat{\boldsymbol{X}}(s, \overline{\boldsymbol{\xi}})
\end{aligned}
$$

where the reduced order matrices, indicated with the superscript $\wedge$, can be calculated by means of a suitable projection matrix $\boldsymbol{F}$ as:

$$
\begin{aligned}
\widehat{\boldsymbol{C}}(\overline{\boldsymbol{\xi}}) & =\boldsymbol{F}^{T} \boldsymbol{C}(\overline{\boldsymbol{\xi}}) \boldsymbol{F} \\
\widehat{\boldsymbol{G}}(\overline{\boldsymbol{\xi}}) & =\boldsymbol{F}^{T} \boldsymbol{G}(\overline{\boldsymbol{\xi}}) \boldsymbol{F} \\
\widehat{\boldsymbol{B}}(\overline{\boldsymbol{\xi}}) & =\boldsymbol{F}^{T} \boldsymbol{B}(\overline{\boldsymbol{\xi}}) \\
\widehat{\boldsymbol{L}}(\overline{\boldsymbol{\xi}}) & =\boldsymbol{F}^{T} \boldsymbol{L}(\overline{\boldsymbol{\xi}})
\end{aligned}
$$

The projection matrix $\boldsymbol{F}$ can be calculated using a MOR technique, such as the Krylov-based Laguerre-SVD [27] or PRIMA [28] algorithms. Therefore, it is possible to calculate for each combination of values of the random variables $\xi$ in the stochastic space $\Omega$ the corresponding reduced system in a descriptor state-space form. Let us suppose that we have calculated $K$ set of reduced matrices $\left[\widehat{\boldsymbol{C}}_{k}, \widehat{\boldsymbol{G}}_{k}, \widehat{\boldsymbol{B}}_{k}, \widehat{\boldsymbol{L}}_{k}\right]_{k=1}^{K}$ with common dimension for the corresponding values of the random variables $\left[\boldsymbol{\xi}_{k}\right]_{k=1}^{K}$ (initial sampling) in the stochastic space $\Omega$. To evaluate the common order for all the $K$ reduced models that will lead to accurate results, first we calculate a reduced order model only for the set of $U$ corner points $\left[\boldsymbol{\xi}_{u}\right]_{u=1}^{U}$ where $U \subset K$, aiming at minimizing the error with respect to the system frequency response $\boldsymbol{H}\left(s, \boldsymbol{\xi}_{u}\right)$ calculated with the original large dimension system matrices $\boldsymbol{C}\left(\boldsymbol{\xi}_{u}\right), \boldsymbol{G}\left(\boldsymbol{\xi}_{u}\right), \boldsymbol{B}\left(\boldsymbol{\xi}_{u}\right), \boldsymbol{L}\left(\boldsymbol{\xi}_{u}\right)$, for $u=1, \ldots, U$. Next, we compute the corresponding set of $K$ projection matrices of common order $\boldsymbol{F}_{k}$ for $k=1, \ldots, K$. Finally, all the projection matrices calculated so far are stacked in a projection matrix as

$$
\boldsymbol{F}_{\text {Union }}=\left[\boldsymbol{F}_{1}, \boldsymbol{F}_{2}, \ldots, \boldsymbol{F}_{K}\right]
$$

The accuracy of the $K$ reduced models with common order, which is estimated by using the $U$ corner points, can be verified by comparing the corresponding frequency responses with respect to the system frequency responses calculated using the $K$ original large dimension system matrices. If, for a particular example, the desired accuracy can not be achieved by using only the $U$ corner points for the order evaluation, it is always possible to estimate the common order using all the $K$ initial samples. However, the choice of using corners to estimate the common order has proven to be accurate in many cases and allows to save computational time [20].

Now, it is necessary to compute a common projection matrix to be able to calculate a parametric reduced order model over the design points $\left[\boldsymbol{\xi}_{k}\right]_{k=1}^{K}$. This goal is achieved in two steps: first the SVD decomposition of the projection matrix $\boldsymbol{F}_{\text {Union }}$ is calculated as:

$$
\boldsymbol{U} \boldsymbol{\Sigma} \boldsymbol{V}^{T}=\operatorname{svd}\left(\boldsymbol{F}_{\text {Union }}\right)
$$

Second, to guarantee the compactness of the common projection matrix it can be defined a common reduced order $r$ based on the first $r$ significant singular values, where the individuation of the desired $r$ significant values can be performed by setting a threshold to the ratio of the singular values with respect to the largest singular value. Indeed, a common projection matrix $\boldsymbol{Q}_{C}$ can now be expressed as:

$$
\boldsymbol{Q}_{C}=\boldsymbol{U}_{r}
$$

where $\boldsymbol{U}_{r}$ is the matrix $\boldsymbol{U}$ resulting from the SVD decomposition (31) for the first $r$ significant singular values. Hence, the desired reduced order matrices with common order can be expressed as:

$$
\begin{aligned}
\tilde{\boldsymbol{C}}_{k}\left(\boldsymbol{\xi}_{k}\right) & =\boldsymbol{Q}_{C}^{T} \boldsymbol{C}\left(\boldsymbol{\xi}_{k}\right) \boldsymbol{Q}_{C} \\
\tilde{\boldsymbol{G}}_{k}\left(\boldsymbol{\xi}_{k}\right) & =\boldsymbol{Q}_{C}^{T} \boldsymbol{G}\left(\boldsymbol{\xi}_{k}\right) \boldsymbol{Q}_{C} \\
\tilde{\boldsymbol{B}}_{k}\left(\boldsymbol{\xi}_{k}\right) & =\boldsymbol{Q}_{C}^{T} \boldsymbol{B}\left(\boldsymbol{\xi}_{k}\right) \\
\tilde{\boldsymbol{L}}_{k}\left(\boldsymbol{\xi}_{k}\right) & =\boldsymbol{Q}_{C}^{T} \boldsymbol{L}\left(\boldsymbol{\xi}_{k}\right)
\end{aligned}
$$

for $k=1, \ldots, K$, where the superscript $\sim$ represents the reduced matrices with common order.

Finally, the PC model for the matrices $\tilde{\boldsymbol{C}}, \tilde{\boldsymbol{G}}, \tilde{\boldsymbol{B}}, \tilde{\boldsymbol{L}}$ can be computed. First of all, it is necessary to calculate the basis functions $\left[\varphi_{i}\right]_{i=0}^{M}$ following the approaches indicated in Section II. Without loss of generality, let us suppose that the random variables $\boldsymbol{\xi}$ are independent. Hence, the number of 
basis function $M+1$ can be chosen upfront according to (3) considering that $P$ can be limited between two and five [4], [24], [26] for practical applications. Finally, the PC coefficient matrices of $\tilde{\boldsymbol{C}}, \tilde{\boldsymbol{G}}, \tilde{\boldsymbol{B}}, \tilde{\boldsymbol{L}}$ of (33)-(36) can be calculated by means of the linear regression approach (see Section II). The linear regression approach calculates the desired PC coefficients solving a suitable over-determined least-square system [7], that for the reduced state-space matrix $\tilde{\boldsymbol{C}}$ can be written as

$$
\Psi \alpha=R
$$

where $\boldsymbol{\Psi} \in \mathbb{R}^{K \tilde{Z} \times(M+1) \tilde{Z}}, \boldsymbol{\alpha} \in \mathbb{R}^{(M+1) \tilde{Z} \times \tilde{Z}}, \boldsymbol{R} \in \mathbb{R}^{K \tilde{Z} \times \tilde{Z}}$ and $\tilde{Z}$ represents the order of the matrix $\tilde{C}$. In particular, the $k$-th row of the matrix $\Psi$ contains the multivariate polynomial basis functions $\varphi_{i}$ for $i=0, \ldots, M$ evaluated in $\boldsymbol{\xi}_{k}$ for $k=1, \ldots, K$ multiplied by the identity matrix of the same dimension as the matrix $\tilde{\boldsymbol{C}}$. The corresponding set of values of the matrix $\tilde{\boldsymbol{C}}_{k}\left(\boldsymbol{\xi}_{k}\right)$ for $k=1, \ldots, K$ are stored in the matrix $\boldsymbol{R}$. Finally, the desired PC coefficients $\tilde{\boldsymbol{C}}_{i}$ for $i=0, \ldots, M$ are collected in the matrix $\boldsymbol{\alpha}$. Equation (37) for the each descriptor state-space matrices can be solved in a least squares sense using an element-wise, columnwise or matrix-wise approach.

Since the linear regression approach requires to solve an over-determined linear system in the form (37), the number of initial samples $K$ is chosen according to the following relation [7]

$$
K \approx 2(M+1)
$$

Note that, equation (37) can be solved in a least squares sense using an element-wise, column-wise or matrix-wise approach.

At this point we have calculated a PC model of the reduced descriptor state-space matrices in the form (9). For example, the matrix $\tilde{\boldsymbol{C}}(\boldsymbol{\xi})$ can be written as

$$
\tilde{\boldsymbol{C}}(\boldsymbol{\xi}) \approx \sum_{i=0}^{M} \tilde{\boldsymbol{C}}_{i} \varphi_{i}(\boldsymbol{\xi})
$$

Finally, it is possible to write two equations in the form (10) and (11) for the reduced order descriptor state-space matrices. Applying the same procedure discussed above, it is possible to compute a frequency-dependent linear system in the form (15), but the overall dimension of this system is much smaller than the corresponding one related to the use of the original large scale matrices. Therefore, the PC expansion of the system transfer function can be calculated following the same procedure described above (see equations (16)-(21)).

The proposed technique is flexible, since the transfer function $\boldsymbol{H}$ of a generical multiport system can be expressed by different representations (e.g. scattering or admittance parameters), it allows to use different MOR techniques to calculate the reduced systems, and it offers a reduced computational complexity with respect to the previous approaches [11][13]. The novel proposed technique allows to perform the VA of large dimension systems, such as the ones resulting from EM simulators, with accuracy and efficiency, thanks to the expression of the system transfer function as a suitable combination of PC expansion and MOR methods. Finally, the proposed method does not require to compute a PC model of the projection operator. The techniques [11]-[13] assume implicitly that the projection operator as a function of the parameters chosen for the variability analysis $\boldsymbol{\xi}$ can be accurately modeled by a PC expansion. However, in the authors' experience, the calculation of a PC model of the projection operator can be prone to inaccuracies, when the system under study is quite sensitive to the parameters chosen for the variability analysis or when the range of variation of these parameters is large enough. The projection operator is computed independently for each initial sample in the stochastic space and it may not result smooth enough as a function of the stochastic parameters $\xi$ to be accurately modeled by a PC model. Section IV illustrates this aspect.

However, the proposed technique has a limitation: it can still be applied if the number of uncertain parameters is relatively large (i.e. $N=10$ ), but at the cost of a loss of efficiency. Indeed, the number $K$ of initial samples is chosen according to (38), and the number of basis function $M+1$ increases rapidly with the number of uncertain parameter $N$, according with (3). Therefore, the calculation of the $K$ initial samples of the large scale system can be expensive, and the computational costs of (31) and of solving the reduced system in the form (15) can increase as well. This limitation is originated by the formulation of the PC expansion: the number $M+1$ of basis functions of any PC model in the form (9) increases rapidly with the number of uncertain parameter $N$, according to (3). Therefore, the corresponding number of $M+1$ unknown PC coefficients that must be estimated is large.

The flowchart of the proposed approach is shown in Fig. 1.

\section{NUMERICAL EXAMPLES}

In this Section, we show the results of the VA performed with the proposed technique for two different structures. In each example, the random variables in the vector $\boldsymbol{\xi}$ are assumed independent and with uniform PDF. The corresponding basis functions are products of the Legendre polynomials [5]. The scalar products resulting from the use of the Galerkin projections are calculated analytically beforehand.

The validation of the accuracy and efficiency of the proposed technique is performed by means of a comparison with the results of other VA techniques. The simulations are performed using MATLAB ${ }^{1}$ R2012a on a Windows platform equipped with an Intel Core2 Extreme CPU Q9300 $2.53 \mathrm{GHz}$ and 8 GB RAM.

\section{A. Transmission line}

In this first example, the scattering parameters of two coupled uniform microstrip lines are considered as a stochastic process with respect to the length of the line in the frequency range $[100 \mathrm{kHz}-3 \mathrm{GHz}]$. The cross section of the microstrip lines is shown in Fig. 2.

The frequency independent per-unit-length parameters of the

\footnotetext{
${ }^{1}$ The Mathworks, Inc., Natick
} 


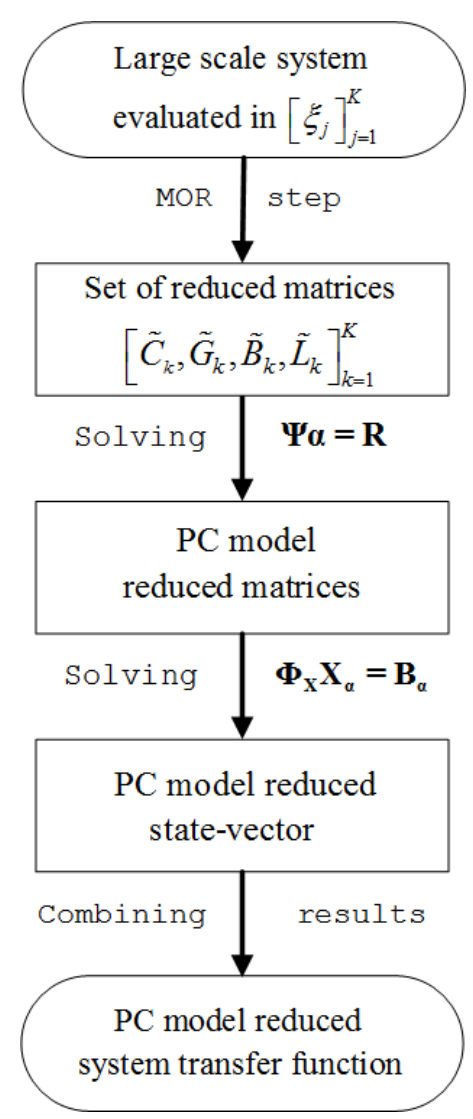

Fig. 1. Flowchart of the proposed modeling strategy.

lines are [29]

$$
\begin{aligned}
\boldsymbol{R}_{p u l} & =\left[\begin{array}{cc}
0.2 & 0 \\
0 & 0.2
\end{array}\right] \frac{\Omega}{\mathrm{m}} \\
\boldsymbol{L}_{p u l} & =\left[\begin{array}{ll}
0.28 & 0.07 \\
0.07 & 0.28
\end{array}\right] \frac{\mathrm{nH}}{\mathrm{m}} \\
\boldsymbol{G}_{p u l} & =\left[\begin{array}{ll}
0 & 0 \\
0 & 0
\end{array}\right] \frac{\mathrm{S}}{\mathrm{m}} \\
\boldsymbol{C}_{p u l} & =\left[\begin{array}{ll}
0.122 & -0.05 \\
-0.05 & 0.122
\end{array}\right] \frac{\mathrm{pF}}{\mathrm{m}}
\end{aligned}
$$

Starting from the per-unit-length parameters, the corresponding matrices for the admittance representation are computed using the segmentation method described in [27] by dividing the lines in 1650 sections of equal length, which gives state-space matrices of order 6602. Then, these matrices are converted into the corresponding ones for the scattering representation as in [30]. The corresponding descriptor state-

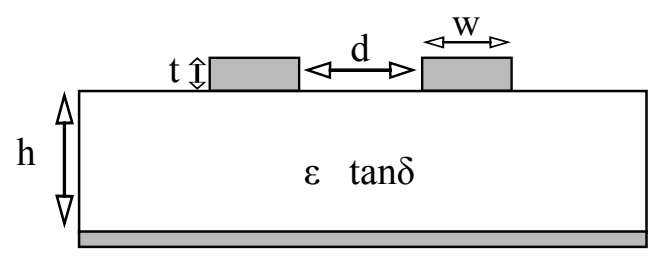

Fig. 2. Example A. Cross section of the coupled microstrips. space representation is

$$
\begin{aligned}
(s \boldsymbol{C}+\boldsymbol{G}) \boldsymbol{X}(s) & =\boldsymbol{B} \\
\boldsymbol{H}(s) & =\boldsymbol{L}^{T} \boldsymbol{X}(s)+\boldsymbol{D}
\end{aligned}
$$

where the matrix $\boldsymbol{D}$ is the identity matrix of dimension $N_{p} \times$ $N_{p}$.

It is important to notice that, the scattering parameters of the two coupled microstrips can be efficiently computed using the exact transmission line theory, therefore the VA could be performed without the calculation of a large descriptor state-space model. However, the calculation of a state-space representation of order 6602 allows to verify the performances of the novel proposed method for the case in which the SVD decomposition (31) is computed for a very high dimension matrix. Finally, the VA of the system in Fig. 2 is performed with respect to the length of the lines in two different ranges of variations and for different orders of the PC models. The performances of the proposed method are compared with the results of the technique [13], for the same number of initial length samples. Note that, the reduced model in the form (16) of [13] are calculated by means of the Galerkin projections instead of truncating the corresponding expansion, since the order of the PC models used in this example is greater than one.

First, the length of the lines varies within the range $[9.75-10.25] \mathrm{cm}$ with a nominal value $L_{0}=10 \mathrm{~cm}$ as a uniform random variable. The corresponding PC expansion is calculated using $P=2$ and $M=2$, according to (3). Therefore, the descriptor state-space form (44) and (45) is computed over a regular grid of $K=6$ samples. The MOR technique PRIMA [28] is used to calculate the reduced order model, and a maximum absolute model error of $-50 \mathrm{~dB}$ over 80 frequency samples is targeted to estimate the common order of the reduced models. To build the common projector matrix, 0.0001 is chosen as threshold to individuate the first $r$ significant singular values of the SVD decomposition in (31) leading to a common projection matrix $\boldsymbol{Q}_{C} \in \mathbb{R}^{6602 \times 64}$. Finally, $K$ reduced models in a descriptor state-space form of order 64 are computed and modeled using PC expansions as previously discussed.

The Table I shows the total computational time of the proposed technique detailing the cost of the different operations. In particular, the element "Initial Data" in Table I represents the operation of calculating the descriptor state-space matrices for the chosen length samples and the corresponding scattering parameters for the two corner points. It is important to specify that, the latter operation takes $1 \mathrm{~h} 11 \mathrm{~min} 9.7 \mathrm{~s}$, while the calculation of the descriptor state-space matrices for all the six length samples requires only $29 \mathrm{~min} 24.8 \mathrm{~s}$. Hence, a MC analysis performed calculating the scattering parameters using the corresponding descriptor state-space models would require approximately 281d $3 \mathrm{~h} \mathrm{10min} \mathrm{33.3s,} \mathrm{considering} 10000$ length samples. Therefore, to validate the results of the VA for this example, the MC analysis is performed using the exact transmission line theory. The element "Projection Operator" in Table I indicates the calculation of the projection operator with common order for all the initial length samples, while the 
element "Reduced DSS Matrices" represents the calculation of the reduced matrices in the form $(33)-(36)$, where the symbol DSS stands for descriptor state-space. Note that, the latter operation requires only few seconds, even if the initial descriptor state-space has order 6602. Finally, the elements in the last three rows of Table I indicate the calculation of the PC model of the reduced descriptor state-space matrices using the linear regression method, the computation of the PC model of the scattering parameters solving an augmented linear system for the reduced state-space matrices in the form (15), and the computation of the mean and the variance of the magnitude of the scattering parameters using the corresponding PC model, respectively.

TABLE I

EXAmple A. COMPUTATIONAL TIME OF THE PROPOSED PC-BASED TECHNIQUE FOR $L \in[9.75-10.25] \mathrm{CM}$

\begin{tabular}{|c|c|}
\hline Technique & Computational time \\
\hline \hline Proposed PC-based Method & $2 h 11$ min $4.9 \mathrm{~s}$ \\
\hline \hline Detailed Description of the Computational Time & \\
\hline Initial Data & $1 h 40 \min 34.5 \mathrm{~s}$ \\
\hline Projection Operator & $30 \min 22.6 \mathrm{~s}$ \\
\hline Reduced DSS Matrices & $3.2 \mathrm{~s}$ \\
\hline PC Model Reduced DSS Matrices & $0.9 \mathrm{~s}$ \\
\hline PC Model S-param & $0.8 \mathrm{~s}$ \\
\hline Mean and Variance S-param & $2.9 \mathrm{~s}$ \\
\hline
\end{tabular}

Table II shows the computational time for the VA performed with the technique [13], in a similar form of Table I. Again, to estimate the common order of the projection operators calculated for all the initial length samples $-50 \mathrm{~dB}$ is assumed as maximum absolute model error. Note that, the element "PC Model Initial DSS Matrices and Proj. Op." in Table II describes the calculation through numerical integration of the PC model of the initial descriptor state-space matrices and of the projection operator.

In this case, the novel proposed method is more efficient in terms of computational time with respect to the technique [13]. Indeed, the calculation of the SVD decomposition of the projection operator (31) is much more efficient than the calculation of the PC model of the initial descriptor state-space matrices and projection operator required by [13], see Tables I and II, even if just three PC coefficients must be estimated via numerical integration for [13].

Furthermore, the novel proposed PC method is much more efficient in terms of memory requirements, as shown in Table III. As memory requirements we indicate the amount of Mbytes that are necessary to load a particular matrix in the
TABLE II

EXAmple A. Computational time of the teChNiQue [13] FoR

$$
L \in[9.75-10.25] \mathrm{CM}
$$

\begin{tabular}{|c|c|}
\hline Technique & Computational time \\
\hline \hline PC-based Technique [13] & $2 h 17$ min $0.3 \mathrm{~s}$ \\
\hline Detailed Description of the Computational Time & \\
\hline Initial Data & $1 \mathrm{~h} 40 \mathrm{~min} 29.6$ \\
\hline Projection Operator & $30 \min 18 \mathrm{~s}$ \\
\hline PC Model Initial DSS Matrices and Proj. Op. & $5 \min 54.1 \mathrm{~s}$ \\
\hline PC Model Reduced DSS Matrices & $14.5 \mathrm{~s}$ \\
\hline PC Model S-param & $1.1 \mathrm{~s}$ \\
\hline Mean and Variance S-param & $3 \mathrm{~s}$ \\
\hline
\end{tabular}

TABLE III

EXAMPLE A. MEMORY REQUIREMENTS OF THE PROPOSED PC-BASED TECHNIQUE AND OF THE TECHNIQUE [13] FOR $L \in[9.75-10.25]$ CM

\begin{tabular}{|c|c|c|}
\hline Elements & New technique & Technique [13] \\
\hline Original DSS Matrices & 3991.67 Mbytes & 3991.67 Mbytes \\
\hline Projection Operator & 16.92 Mbytes & 16.92 Mbytes \\
\hline $\begin{array}{l}\text { Common Proj. Op. (32) and } \\
\text { Reduced DSS Matrices (33)-(36) }\end{array}$ & 3.23 Mbytes & \\
\hline $\begin{array}{l}\text { PC Model Initial DSS Matrices } \\
\text { and Projection Operator }\end{array}$ & 1.58 Mbytes & 1.03 Mbytes \\
\hline \multicolumn{2}{|c|}{ PC Model Reduced Matrices } & \\
\hline
\end{tabular}

computer RAM. In particular, the memory necessary to store the descriptor state-space matrices for all the initial length samples and the corresponding projection operators is the same for both the techniques, see the elements "Original DSS Matrices" and "Projection Operator" in Table III, while the computation of the reduced matrices (33)-(36) using the common projection operator (32) is much more efficient than the calculation of the PC models of the descriptor state-space matrices and of the projection operator, see elements "Common Proj. Op. (32) and Reduced DSS Matrices (33)-(36)" and "PC Model Initial DSS Matrices and Projection Operator" in Table III, respectively.

Finally, the proposed PC-based stochastic model order reduction technique shows an excellent accuracy compared with the MC analysis, performed over $10000 L$ samples, in 
computing system variability features, as shown in Figs. 3 - 4. In particular, Figs. 3 - 4 show the mean and standard deviation of the magnitude of the element $S_{32}$ obtained with the MC analysis, the proposed PC method and the technique [13]. Similar results can be obtained for the other entries of the scattering matrix.
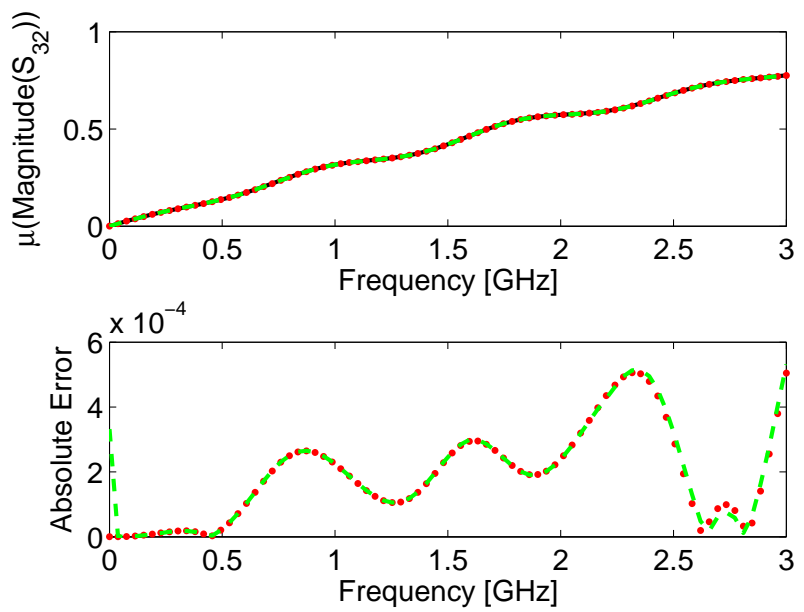

Fig. 3. Example A. The top plot shows a comparison between the mean of the magnitude of $S_{32}$ obtained with the MC analysis (full black line), the proposed PC-based method (red dotted line) and the technique [13] (green dashed line). The lower plot shows the absolute error of the two PC-based VA techniques with respect to the MC analysis.
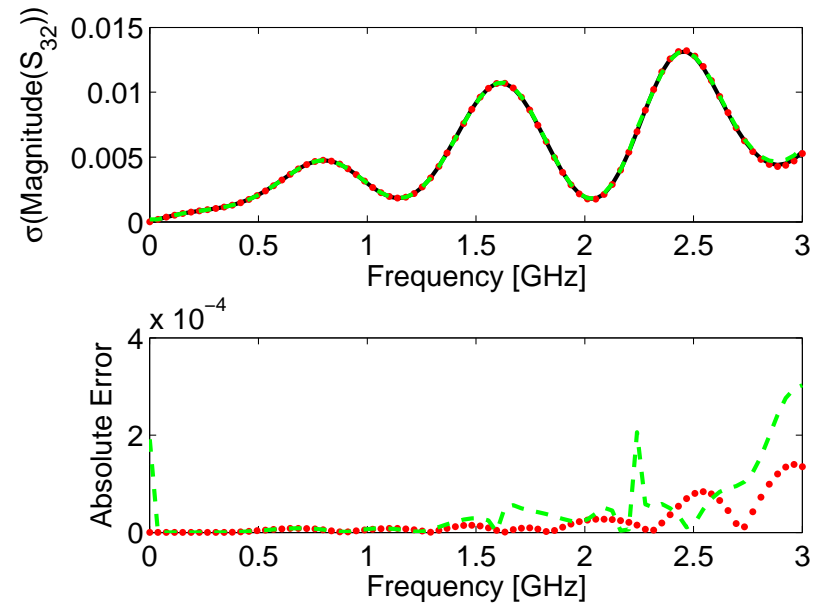

Fig. 4. Example A. The top plot shows a comparison between the standard deviation of the magnitude of $S_{32}$ obtained with the MC analysis (full black line), the proposed PC-based method (red dotted line) and the technique [13] (green dashed line). The lower plot shows the absolute error of the two PCbased VA techniques with respect to the MC analysis.

Next, the lines length is considered as a uniform random variable varying in the range $[9-11] \mathrm{cm}$. An example of the scattering parameters variability with respect to the chosen random variable is given in Fig. 5. The reduced order models are calculated again using the MOR technique PRIMA [28], targeting an absolute model error of $-50 \mathrm{~dB}$ over 80 frequency samples. The common projector matrix, obtained choosing
0.0001 as threshold to individuate the first $r$ significant singular values of the SVD decomposition in (31), has order $\boldsymbol{Q}_{C} \in \mathbb{R}^{6602 \times 72}$.

The proposed PC-based stochastic model order reduction technique is more efficient both in terms of memory and computational requirements with respect to technique [13], see Tables IV - VI, and it shows a great accuracy compared with the classical MC analysis, performed over $10000 L$ samples, in computing system variability features, as shown in Figs. 6 - 8. In particular, Figs. 6 - 7 show the mean and standard deviation of the magnitude of the element $S_{43}$ obtained with the MC analysis, the proposed PC method and the technique [13]. Similar results can be obtained for the other entries of the scattering matrix.

TABLE IV

EXAmple A. Computational time of THE PROPOSED PC-BASED TECHNIQUE FOR $L \in[9-11] \mathrm{CM}$

\begin{tabular}{|c|c|}
\hline Technique & Computational time \\
\hline Proposed PC-based Method & $2 h 32 \mathrm{~min} 14.3 \mathrm{~s}$ \\
\hline Detailed Description of the Computational Time & \\
\hline Initial Data & $1 \mathrm{~h} 50 \mathrm{~min} 0.3 \mathrm{~s}$ \\
\hline Projection Operator & $42 \min 2.6 \mathrm{~s}$ \\
\hline Reduced DSS Matrices & $4.8 \mathrm{~s}$ \\
\hline PC Model Reduced DSS Matrices & $1.7 \mathrm{~s}$ \\
\hline PC Model S-param & $1.5 \mathrm{~s}$ \\
\hline Mean and Variance S-param & $3.4 \mathrm{~s}$ \\
\hline
\end{tabular}

It is important to notice that, in this case, the results of the VA performed using the technique [13] are inaccurate with respect to the $\mathrm{MC}$ analysis performed using the transmission lines theory over $10000 \mathrm{~L}$ samples, see Figs. 6 - 7. Indeed the calculation via numerical integration of the PC model of the projection operator is inaccurate, see Fig. 9. It is worth to notice that, the calculation of the PC model of the original descriptor state-space matrices and of the projection operator is two times more expensive with respect to the previous case, see Tables II and V, and it requires much more memory, see Tables III and VI.

\section{B. Bended Conductors}

In this second example, a system of three bended conductors in free space has been modeled. Its layout is shown in Fig. 10. The copper conductors, placed at a distance of $S_{0}=2$ $\mathrm{mm}$ the one from the other, have width $W_{0}=0.5 \mathrm{~mm}$ and length $L_{0}=5 \mathrm{~mm}$. The copper conductivity is assumed equal to $5.8 \cdot 10^{7} \mathrm{~S} / \mathrm{m}$. 
TABLE V

EXAmple A. Computational time of the technique [13] FoR $L \in[9-11] \mathrm{CM}$

\begin{tabular}{|c|c|}
\hline Technique & Computational time \\
\hline PC-based Technique [13] & $2 h 45 \min 51.2 \mathrm{~s}$ \\
\hline \hline Detailed Description of the Computational Time & \\
\hline Initial Data & $1 h 49 \min 28.4 \mathrm{~s}$ \\
\hline Projection Operator & $42 \min 24.7 \mathrm{~s}$ \\
\hline PC Model Initial DSS Matrices and Proj. Op. & $13 \min 11.5 \mathrm{~s}$ \\
\hline PC Model Reduced DSS Matrices & $41.8 \mathrm{~s}$ \\
\hline PC Model S-param & $1.3 \mathrm{~s}$ \\
\hline Mean and Variance S-param & $3.5 \mathrm{~s}$ \\
\hline
\end{tabular}

TABLE VI

EXAMPle A. MEMORY REQUiREMENTS OF THE PROPOSED PC-BASED TECHNIQUE AND OF THE TECHNIQUE [13] FOR $L \in[9-11] \mathrm{CM}$

\begin{tabular}{|c|c|c|}
\hline Elements & New technique & Technique [13] \\
\hline Original DSS Matrices & 5322.22 Mbytes & 5322.22 Mbytes \\
\hline Projection Operator & 24.18 Mbytes & 24.18 Mbytes \\
\hline $\begin{array}{l}\text { Common Proj. Op. (32) and } \\
\text { Reduced DSS Matrices (33)-(36) }\end{array}$ & 3.62 Mbytes & \\
\hline $\begin{array}{l}\text { PC Model Initial DSS Matrices } \\
\text { and Projection Operator }\end{array}$ & 2673.2 Mbytes \\
\hline \begin{tabular}{l} 
PC Model Reduced Matrices \\
\hline
\end{tabular} & & 1.48 Mbytes \\
\hline
\end{tabular}

The system admittance parameters are considered as a stochastic process first with respect to the couple of random variables $(L, S)$ varying in a range of $\pm 10 \%$ with respect to the central values previously mentioned. The admittance representation is evaluated in the frequency range [100 kHz $-5 \mathrm{GHz}]$ using the PEEC method [31] over a grid composed of $4 \times 4(L, S)$ samples for the geometrical parameters. The corresponding set of descriptor state-space matrices computed has order 2124 for each initial $(L, S)$ sample. The Laguerre-SVD MOR technique [27] is used to calculate the reduced order models. The evaluation of the common order of the reduced models is performed assuming 0.0001 as maximum weighted rms error between the admittance parameters of the reduced model and the original system over $K_{s}=50$

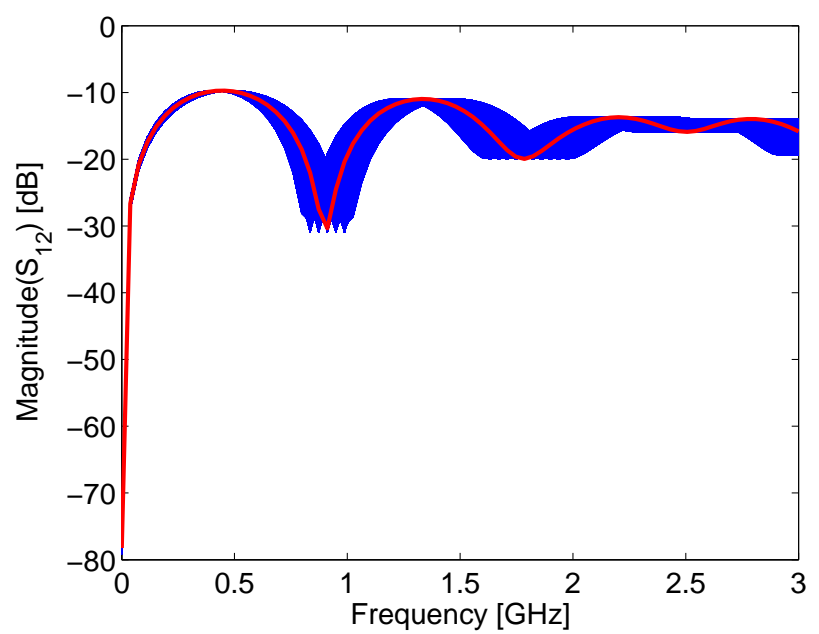

Fig. 5. Example A. Variability of the magnitude of $S_{12}$ calculated for $L \in[9-11]$. The blue lines are the results of the MC simulations and the red thick line corresponds to the nominal value for $L_{0}=10 \mathrm{~cm}$.
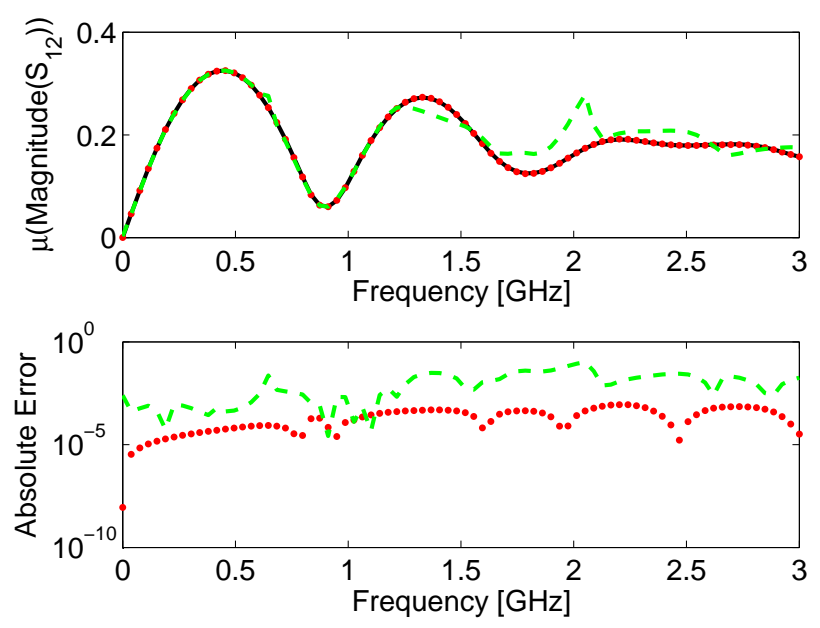

Fig. 6. Example A. The top plot shows a comparison between the mean of the magnitude of $S_{12}$ obtained with the MC analysis (full black line), the proposed PC-based method (red dotted line) and the technique [13] (green dashed line). The lower plot shows the absolute error of the two PC-based VA techniques with respect to the MC analysis in logarithmic scale.

frequency samples:

$$
\begin{aligned}
& \text { Error }_{r m s}= \\
& \sqrt{\frac{\sum_{i=1}^{N_{p}^{2}} \sum_{k=1}^{K_{s}}\left|w_{Y_{i}}\left(s_{k}\right)\left(Y_{r, i}\left(s_{k}\right)-Y_{i}\left(s_{k}\right)\right)\right|^{2}}{N_{p}^{2} K_{s}}}
\end{aligned}
$$

with

$$
w_{Y_{i}}(s)=\left|\left(Y_{i}(s)\right)^{-1}\right|
$$

The evaluation of the significant $r$ singular values of the SVD decomposition in (31) is performed assuming 0.0001 as a threshold. Upon calculation of the common projection matrix (32), each of the $K$ reduced order models calculated for the couple of random variables $(L, S)$ has order 232 . Finally, the set of reduced order matrices is modeled using a second order 

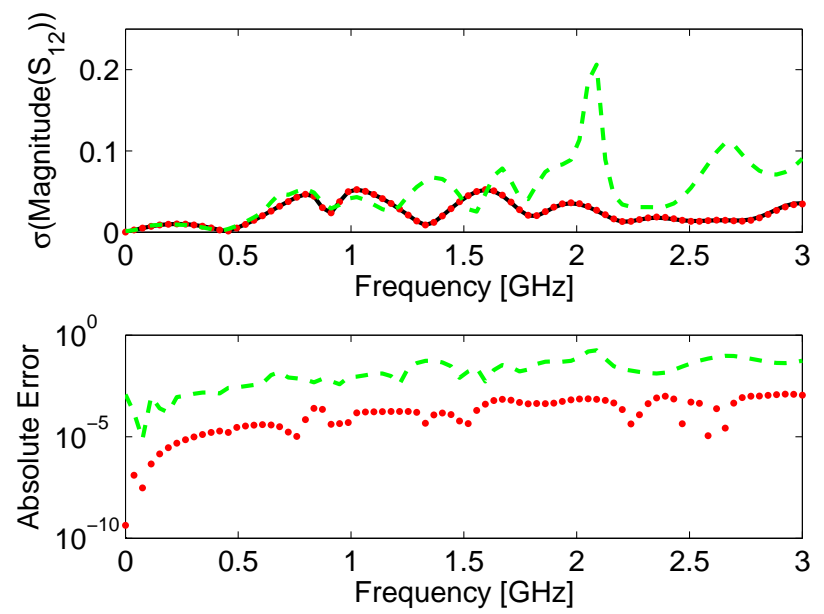

Fig. 7. Example A. The top plot shows a comparison between the standard deviation of the magnitude of $S_{12}$ obtained with the MC analysis (full black line), the proposed PC-based method (red dotted line) and the technique [13] (green dashed line). The lower plot shows the absolute error of the two PCbased VA techniques with respect to the MC analysis in logarithmic scale.

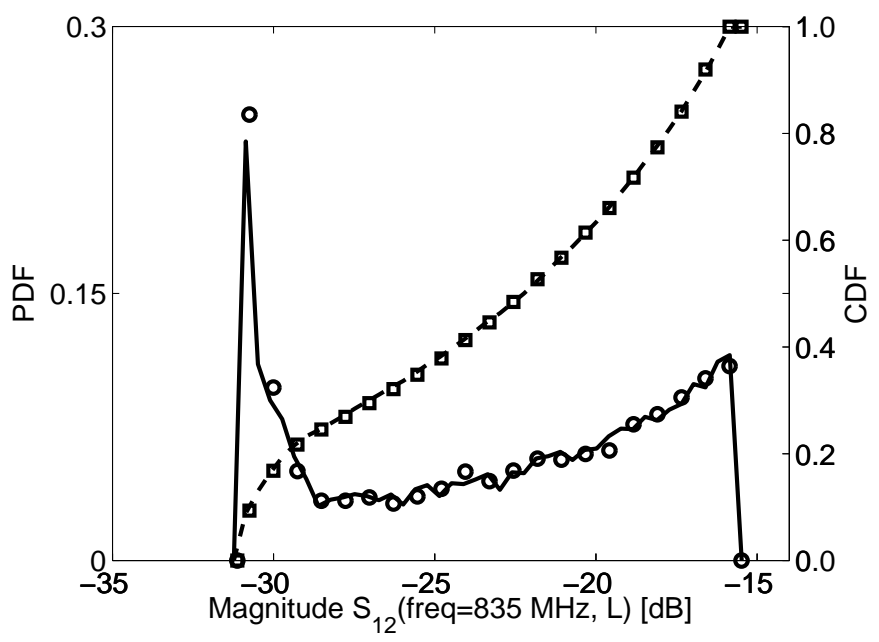

Fig. 8. Example A. PDF and CDF of the magnitude of $S_{12}$ at $835 \mathrm{MHz}$. Full black line: PDF computed using the novel technique; Dashed black line: CDF computed using the novel technique; Circles (o): PDF computed using the MC technique; Squares ( $\square$ ): CDF computed using the MC technique.

$(P=2)$ PC expansion, giving $M=5$ for $(L, S)$, according to $(3)$.

The results of the VA obtained with the novel proposed method are compared with the corresponding ones given by the technique [13] and are validated by means of comparison with results of the MC analysis using the parameterized model order reduction technique [31]. The latter technique is a parameterized model order reduction method that guarantees the overall stability and passivity of parameterized reduced order models, by using passivity preserving interpolation schemes. In particular, first a set of reduced model with the same order are calculated assuming 0.0001 as threshold for the error measure (46). Then, the reduced models obtained are interpolated over the $10000(L, S)$ samples used for the MC analysis. Note that, the parameterized model order reduction technique [31]
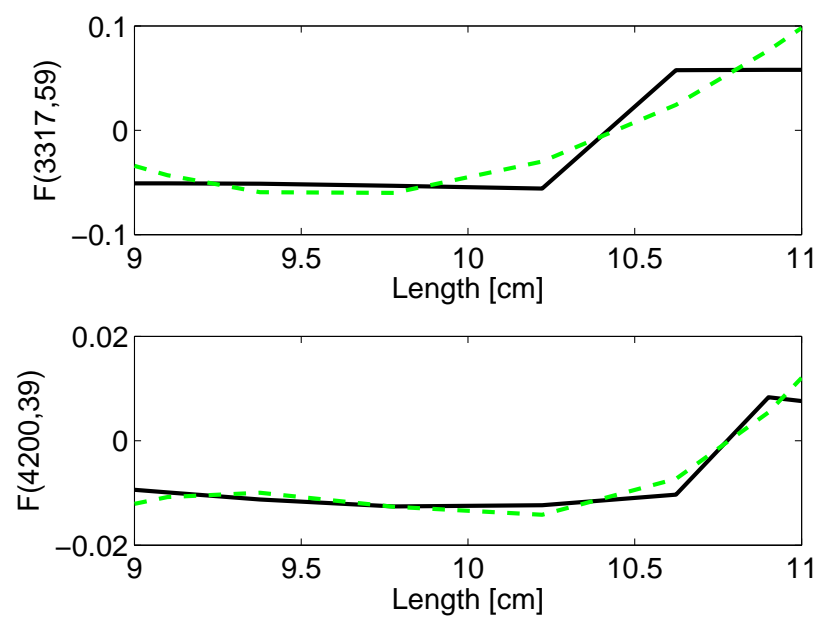

Fig. 9. Example A. The top plot shows a comparison between the value of the 3317th row and 59th column of the projection operator calculated for all the initial length samples (full black line) and the corresponding value obtained using the PC model of the projection operator (green dashed line). The lower plot shows the same comparison for the value of the 4200th row and 39 th column of the projection operator.

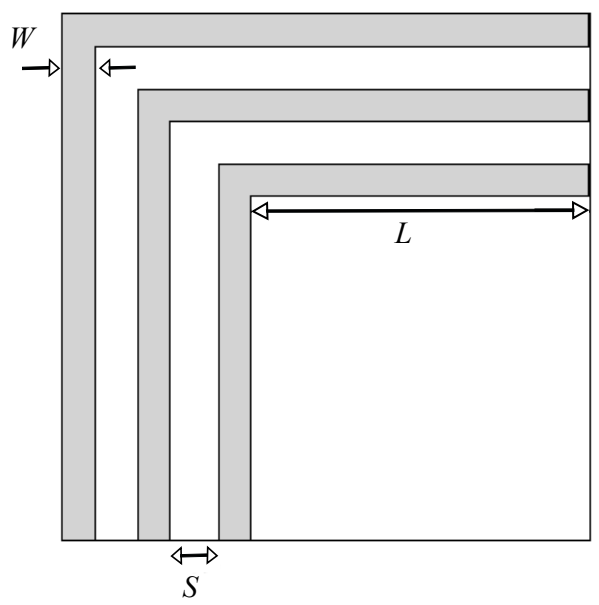

Fig. 10. Example B. Geometry of the system of bended conductors

is applied to the same PEEC matrices calculated for the initial $(L, S)$ samples used for the novel proposed technique. Finally, the initial samples needed by the technique [13] are computed over a Smolyak sparse grid, composed of $29(L, S)$ samples [13], while 0.0001 is assumed as maximum weighted rms error to estimate the common order of the projection operators calculated for all the initial $(L, S)$ samples.

The PC model of the projection operator computed by the technique [13] is not accurate, see Fig. 11. This leads to poor accuracy in computing the system variability features with respect to the corresponding results given by the novel proposed method and the MC analysis performed using the parameterized model order reduction technique [31], as shown in Figs. 12, 13, although the number of initial samples used to implement the technique [13] is almost the double of the ones used for the proposed PC-based method. In particular, Figs. 12 

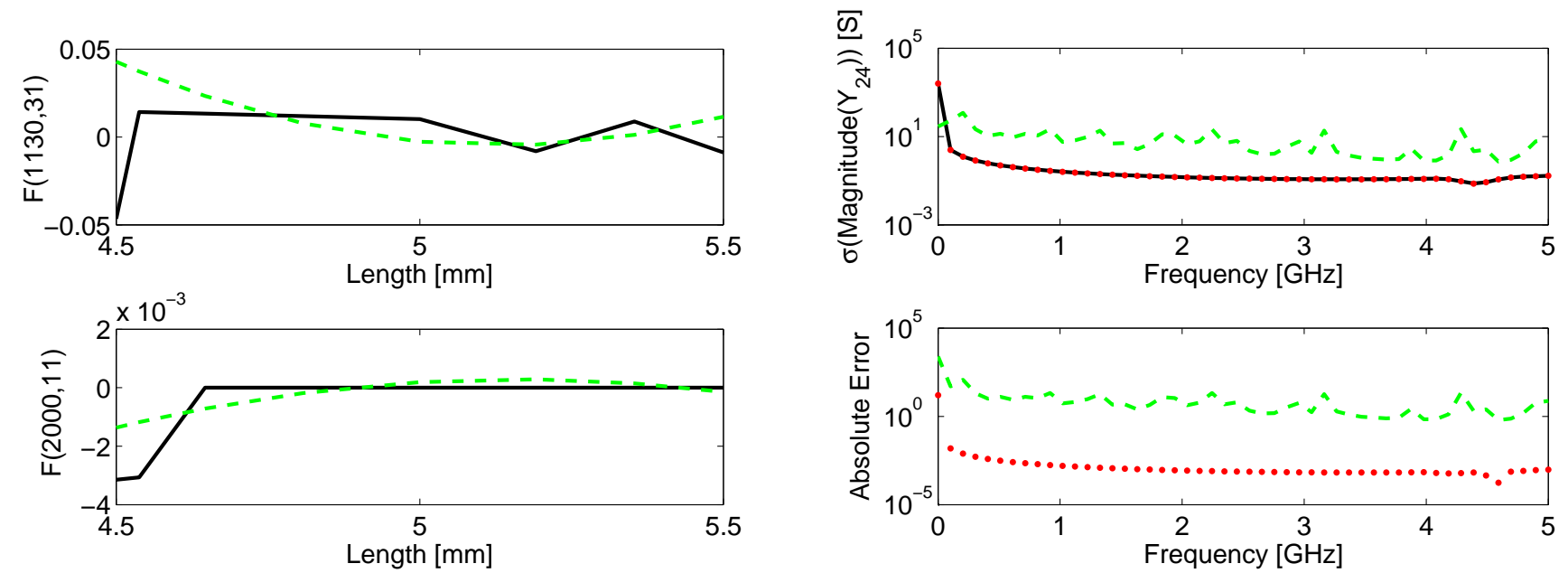

Fig. 11. Example B. The top plot shows a comparison between the value of the 1130th row and 31th column of the projection operator calculated for all the length samples corresponding to the value of spacing $S=2 \mathrm{~mm}$ (full black line) and the corresponding value obtained using the PC model of the projection operator (green dashed line). The lower plot shows the same comparison for the value of the 2000th row and 11th column of the projection operator.

and 13 show the mean and standard deviation of the magnitude of $Y_{24}$ calculated with respect to the random variables $(L, S)$. Similar results can be obtained for the other entries of the admittance parameters. Furthermore, the VA performed with the proposed method shows a great efficiency both in terms of memory and computational time, as shown in Table VII and VIII.

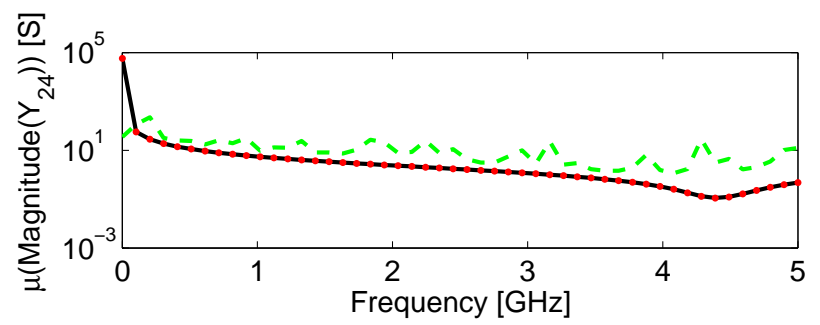

Fig. 13. Example B. Comparison of the standard deviation for the magnitude of $Y_{24}$ with respect to the couple of random variables $(L, S)$ obtained with the MC analysis performed with the technique [31] (full black line), the technique [13] (green dashed line) and the proposed PC-based method (dotted red line).

TABLE VII

EXAMPLE B. EFFICIENCY OF THE PROPOSED PC-BASED TECHNIQUE FOR $(L, S)$

\begin{tabular}{|c|c|}
\hline Technique & Computational time \\
\hline Parameterized model order reduction [31] & $3 \mathrm{~h} 19 \mathrm{~min} 37.6 \mathrm{~s}$ \\
\hline Technique [13] & $1 \mathrm{~h} 25 \mathrm{~min} 23.7 \mathrm{~s}$ \\
\hline Proposed PC-based Method & $47 \min 15.2 \mathrm{~s}$ \\
\hline Detailed Description of the Computational Tim & \\
\hline Initial Data & $45 \min 24 s$ \\
\hline Projection Operator & $1 \min 15 s$ \\
\hline Reduced DSS Matrices & $2.9 \mathrm{~s}$ \\
\hline PC Model Reduced DSS Matrices & $5.7 \mathrm{~s}$ \\
\hline PC Model S-param & $26 s$ \\
\hline Mean and Variance S-param & $1.6 s$ \\
\hline
\end{tabular}

Fig. 12. Example B. Comparison of the mean for the magnitude of $Y_{24}$ with respect to the couple of random variables $(L, S)$ obtained with the MC analysis performed with the technique [31] (full black line), the technique [13] (green dashed line) and the proposed PC-based method (dotted red line).

Finally, the VA of the system in Fig. 10 is performed with respect to the set of random variables $(L, S, W)$, varying in a range of $\pm 10 \%$ with respect to the central values previously mentioned, in order to show the performances of the proposed PC method while increasing the numbers of random variables. Figure 14 shows an example of the system variability with

respect to the chosen random variables $(L, S, W)$. The system admittance parameters are evaluated using the PEEC method over a grid of $3 \times 3 \times 3(L, S, W)$ samples. The evaluation of the common order of the reduced models and of the $r$ significant singular values of the SVD decomposition in (31) is performed with the same setting used in the previous case, giving reduced order models of order 328. Next, the set of reduced order matrices is modeled using a second 
TABLE VIII

EXAmple B. Memory ReQuirements of THE PROPOSED PC-BASED TECHNIQUE AND OF THE TECHNIQUE [13] FOR $(L, S)$

\begin{tabular}{|c|c|c|}
\hline Elements & New technique & Technique [13] \\
\hline Original DSS Matrices & 1102.45 Mbytes & 1998.19 Mbytes \\
\hline Projection Operator & 9.33 Mbytes & 16.92 Mbytes \\
\hline $\begin{array}{l}\text { Common Proj. Op. (32) and } \\
\text { Reduced DSS Matrices (33)-(36) }\end{array}$ & 17.01 Mbytes & \\
\hline $\begin{array}{l}\text { PC Model Initial DSS Matrices } \\
\text { and Projection Operator }\end{array}$ & 5.15 Mbytes & 0.31 Mbytes \\
\hline \begin{tabular}{l} 
PC Model Reduced Matrices \\
\hline
\end{tabular} & & \\
\hline
\end{tabular}
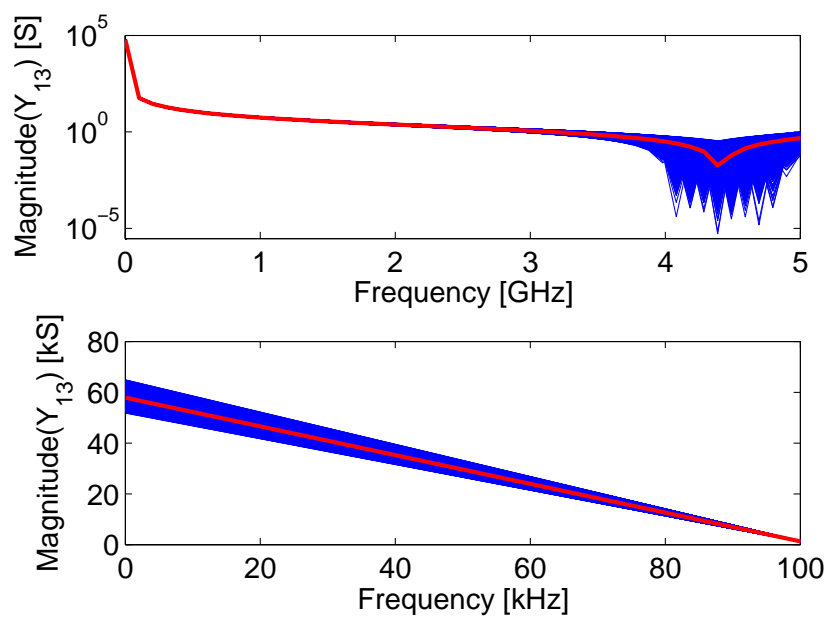

Fig. 14. Example B. The top plot shows the variability of the magnitude of $Y_{13}$ with respect to the random variables $(L, S, W)$ in the frequency range $[100 \mathrm{kHz}-5 \mathrm{GHz}]$. The lower plot shows the variability of the magnitude of $Y_{13}$ in the frequency range $[100 \mathrm{kHz}-100 \mathrm{MHz}]$. In both plots, the blue lines are the results of the MC simulations and the red thick line corresponds to the nominal value for $L_{0}=5 \mathrm{~mm}, S_{0}=2 \mathrm{~mm}$ and $W_{0}=0.5 \mathrm{~mm}$.

order $(P=2)$ PC expansion, giving $M=9$ for $(L, S, W)$, according to (3). The proposed PC-based method is compared with the MC analysis performed with the technique [31] for 100, 1000, 10000 samples.

The proposed method shows a great efficiency in computing the system variability features, as shown in Table IX that compares the computational cost of the MC analysis performed with the technique [31] for 10000 samples and of the novel proposed method, and a great accuracy with respect to the corresponding analysis performed with the technique [31], as described by Figs. 15 - 17. In particular, Figs. 15, 16 show the mean and standard deviation of the magnitude of $Y_{43}$ obtained with the proposed PC-based method and the MC analysis performed using different set of samples. Figure 17 describes the PDF and the CDF of the magnitude of $Y_{11}$ at 100 $\mathrm{kHz}$ calculated with respect to the random variables $(L, S, W)$
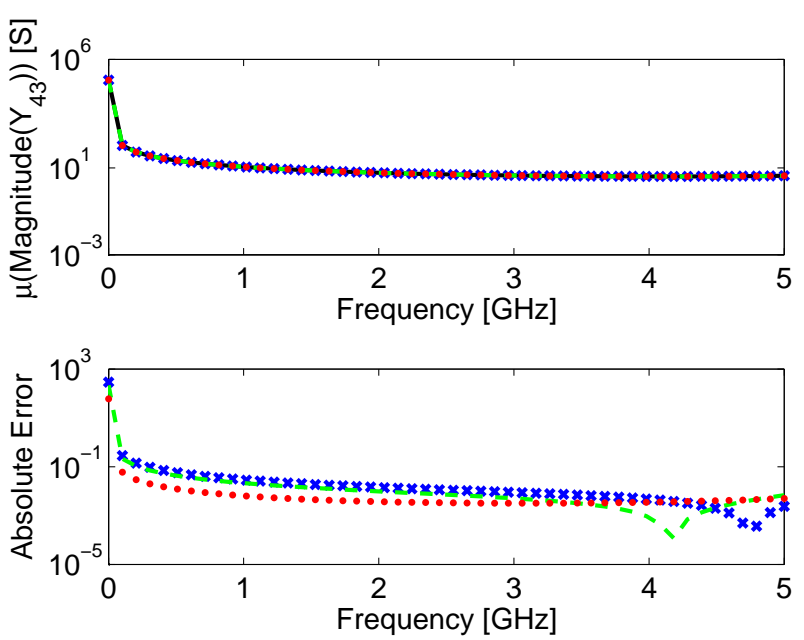

Fig. 15. Example B. Comparison of the mean for the magnitude of $Y_{43}$ obtained with the MC analysis performed with the technique [31] using 10000 (full black line), 1000 (green dashed line) and 100 (blue ex $(\times))(L, S, W)$ samples and the proposed PC-based method (dotted red line).

using 10000 samples for the MC results. Similar results can be obtained for the other entries of the admittance parameters.
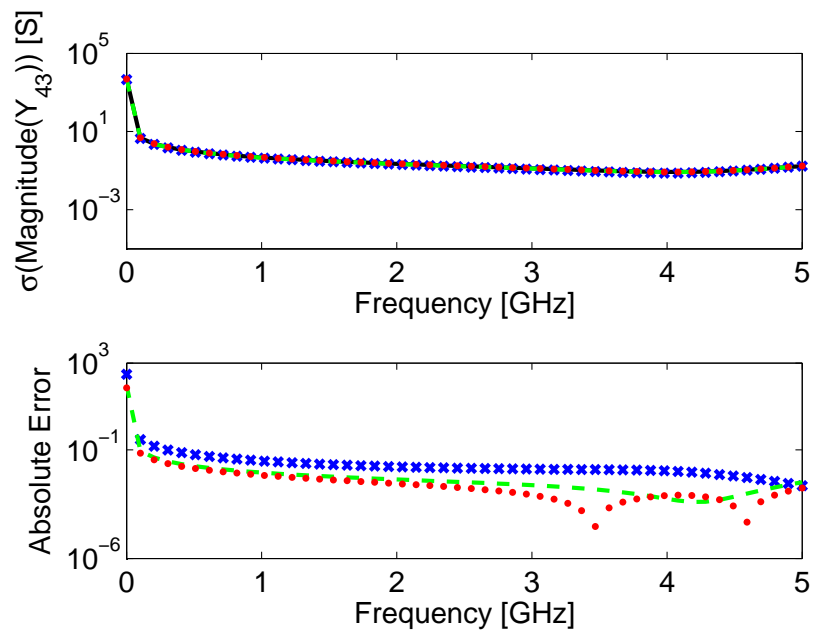

Fig. 16. Example B. Comparison of the standard deviation for the magnitude of $Y_{43}$ obtained with the MC analysis performed with the technique [31] using 10000 (full black line), 1000 (green dashed line) and 100 (blue ex $(\times)$ ) $(L, S, W)$ samples and the proposed PC-based method (dotted red line).

\section{CONCLUSIONS}

In this paper, a novel and efficient technique for stochastic model order reduction of general linear multiport systems is presented. The core of the proposed technique is the application of the PC expansion to a set of reduced order system in a descriptor state-space form obtained through a MOR step. In addition to its accuracy, the proposed approach offers a great flexibility. Indeed, not only the novel method can be applied to systems whose frequency-domain transfer function can be expressed in different forms (e.g. scattering or admittance parameters), but also different MOR techniques can be used for the calculation of the reduced order system in 


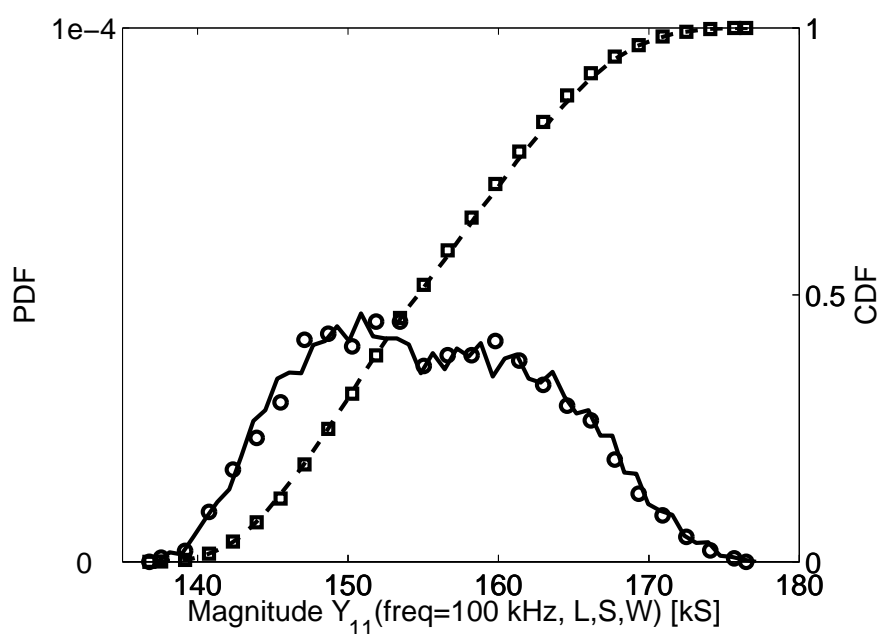

Fig. 17. Example B. PDF and CDF of the magnitude of $Y_{11}$ at $100 \mathrm{kHz}$. Full black line: PDF computed using the novel technique; Dashed black line: CDF computed using the novel technique; Circles (o): PDF computed using the MC technique performed with the technique [31] ; Squares ( $\square$ ): CDF computed using the MC technique performed with the technique [31] .

\section{TABLE IX}

EXAMPLE B. EFFICIENCY OF THE PROPOSED PC-BASED TECHNIQUE FOR

$$
(L, S, W)
$$

\begin{tabular}{|c|c|}
\hline Technique & Computational time \\
\hline Parameterized model order reduction [31] & $6 h$ 1min 42.6 \\
\hline \hline Proposed PC-based Method & $1 \mathrm{~h} 24 \mathrm{~min} 4.5 \mathrm{~s}$ \\
\hline Detailed Description of the Computational Time & \\
\hline Initial Data & $1 \mathrm{~h} 17 \mathrm{~min} 58 \mathrm{~s}$ \\
\hline Calculation Projection Operator & 1 min $55 \mathrm{~s}$ \\
\hline Calculation Reduced DSS Matrices & $7.3 \mathrm{~s}$ \\
\hline PC Model Reduced DSS Matrices & $14.2 \mathrm{~s}$ \\
\hline PC Model S-param & $3 \min 47.9 \mathrm{~s}$ \\
\hline Mean and Variance S-param & $2.1 \mathrm{~s}$ \\
\hline
\end{tabular}

descriptor state-space form. Two distinct pertinent numerical examples validate the accuracy and efficiency of the proposed method with respect to existing techniques concerning the calculation of system variability features.

\section{REFERENCES}

[1] R. F. Harrington, Field Computation by Moment Methods. New York: Macmillan, 1968 .

[2] A. E. Ruehli, "Equivalent circuit models for three dimensional multiconductor systems," IEEE Trans. Microw. Theory Tech., vol. 22, no. 3, pp. 216-221, Mar. 1974.
[3] J. M. Jin, The Finite Element Method in Electromagnetics, 2nd ed. John Wiley and Sons, New York, 2002.

[4] D. Xiu and G.M. Karniadakis, "The Wiener-Askey polynomial chaos for stochastic differential equations," SIAM J. Sci. Comput., vol. 24, no. 2, pp. 619-644, Apr. 2002.

[5] C. Soize and R. Ghanem, "Physical systems with random uncertainties: Chaos representations with arbitrary probability measure," SIAM J. SCI. COMPUT., vol. 26, no. 2, pp. 395-410, Jul. 2004

[6] J. A. S. Witteveen and H. Bijl, "Modeling Arbitrary Uncertainties Using Gram-Schmidt Polynomial Chaos," in Proc. 44th AIAA Aerosp. Sci. Meeting and Exhibit, no. AIAA-2006-0896, Palm Springs, California, Jan. 2006.

[7] M. S. Eldred, "Recent advance in non-intrusive polynomial-chaos and stochastic collocation methods for uncertainty analysis and design," in Proc. 50th AIAA/ASME/ASCE/AHS/ASC Struct., Structural Dynam., Mat. Conf., no. AIAA-2009-2274, Palm Springs, California, May 2009.

[8] G. Blatman and B. Sudret, "An adaptive algorithm to build up sparse polynomial chaos expansions for stochastic finite element analysis," Probabilistic Engineering Mechanics, vol. 25, no. 2, pp. 183 - 197, 2010.

[9] S. Vrudhula, J.M. Wang, and P. Ghanta, "Hermite polynomial based interconnect analysis in the presence of process variations," IEEE Trans. Comput.-Aided Design Integr. Circuits Syst., vol. 25, no. 10, pp. 20012011, 2006.

[10] N. Mi, S. X.-D Tan, Y. Cai, and X. Hong , "Fast variational analysis of on-chip power grids by stochastic extended Krylov subspace method," IEEE Trans. Comput.-Aided Design Integr. Circuits Syst., vol. 27, no. 11, pp. 1996-2006, 2008.

[11] P. Sumant, H. Wu, A. Cangellaris, N. Aluru, "A sparse grid based collocation method for model order reduction of finite element approximations of passive electromagnetic devices under uncertainty," in Proc. Microwave Symposium Digest (MTT), 2010 IEEE MTT-S International, Anaheim, California, May 2010.

[12] P. Sumant, H. Wu, A. Cangellaris, and N. Aluru, "Order reduction of finite element models of passive electromagnetic structures with statistical variability," in Electromagnetic Theory (EMTS), 2010 URSI International Symposium on, Aug. 2010, pp. 688 -691.

[13] P. Sumant, H. Wu, A. Cangellaris and N. Aluru, "Reduced-order models of finite element approximations of electromagnetic devices exhibiting statistical variability," IEEE Trans. Antennas Propag., vol. 60, no. 1, pp. 301-309, Jan. 2012.

[14] L. Knockaert, T. Dhaene, F. Ferranti, D. De Zutter, "Model order reduction with preservation of passivity, non-expansivity and Markov moments," Systems \& Control Letters, vol. 60, no. 1, pp. 53-61, Jan. 2011.

[15] J. Phillips, L. Daniel, L. M. Silveira, "Guaranteed passive balancing transformations for model order reduction," IEEE Trans. CAD Integr. Circuits Syst., vol. 22, no. 8, pp. 1027-1041, Aug. 2003.

[16] J. Phillips, L. M. Silveira, "Poor man's TBR: a simple model reduction scheme," IEEE Trans. CAD Integr. Circuits Syst., vol. 24, no. 1, pp. 43-55, Jan. 2005.

[17] V. Barthelmann, E. Novak, and K. Ritter, "High dimensional polynomial interpolation on sparse grid," Adv. Comput. Math., vol. 12, no. 4, pp. 273-288, 2000.

[18] E. Novak, and K. Ritter, "High dimensional integration of smooth functions over cubes," Numer. Math., vol. 75, no. 1, pp. 79-97, 1996.

[19] — - "Simple cubature formulas with high polynomial exactness," Construct. Approx., vol. 15, no. 4, pp. 499-522, 1999.

[20] E. R. Samuel, F. Ferranti, L. Knockaert, and T. Dhaene, "Parameterized reduced order models with guaranteed passivity using matrix interpolation," in Signal and Power Integrity (SPI), 2012 IEEE 16th Workshop on, May 2012, pp. 65-68.

[21] A. Der Kiureghian and P. L. Liu, "Structural reliability under incomplete probability information,", J. Eng. Mech., ASCE, vol. 112, no. 1, pp. 85104, 1986.

[22] M. Loéve, Probability Theory, 4th ed. Berlin, Germany: SpringerVerlag, 1977.

[23] A. Papoulis, Probability, Random Variables and Stochastic Processes. Mcgraw-Hill College, 1991.

[24] D. Spina, F. Ferranti, T. Dhaene, L. Knockaert, G. Antonini, and D. Vande Ginste, "Variability analysis of multiport systems via polynomial-chaos expansion," IEEE Trans. Microw. Theory Tech., vol. 60, no. 8, pp. $2329-2338$, Aug. 2012.

[25] D. Vande Ginste, D. De Zutter, D. Deschrijver, T. Dhaene, P. Manfredi, and F. Canavero, "Stochastic modeling-based variability analysis of onchip interconnects," IEEE Trans. Compon., Packag., Manuf. Technol., vol. 2, no. 7, pp. $1182-1192$, Jul. 2012. 
[26] I. S. Stievano, P. Manfredi, F. G. Canavero, "Parameters variability effects on multiconductor interconnects via Hermite polynomial chaos," IEEE Trans. Compon., Packag., Manuf. Technol., vol. 1, no. 8, pp. 12341239, Aug. 2011.

[27] L. Knockaert and D. De Zutter, "Laguerre-SVD reduced order modeling," IEEE Trans. Microw. Theory Tech., vol. 48, no. 9, pp. 1469-1475, Sep. 2000.

[28] A. Odabasioglu, M. Celik, and L. T. Pileggi, "PRIMA: Passive reducedorder interconnect macromodeling algorithm,", IEEE Trans. ComputerAided Design, vol. 17, pp. 645-654, Aug. 1998.

[29] G. Antonini, "A dyadic Green's function based method for the transient analysis of lossy and dispersive multiconductor transmission lines," IEEE Trans. Microw. Theory Tech., vol. 56, no. 4, pp. 880-895, Apr. 2008.

[30] F. Ferranti, G. Antonini, T. Dhaene, and L. Knockaert, "Guaranteed passive parameterized model order reduction of the partial element equivalent circuit (PEEC) method," IEEE Trans. Electromagn. Compat., vol. 52, no. 4, pp. 974-984, Nov. 2010.

[31] F. Ferranti, G. Antonini, T. Dhaene, L. Knockaert, and A.E. Ruehli, "Physics-based passivity-preserving parameterized model order reduction for PEEC circuit analysis," IEEE Trans. Compon., Packag., Manuf. Technol., vol. 1, no. 3, pp. 399-409, Mar. 2011. 